\title{
Review on nanofluids theoretical thermal conductivity models
}

\author{
Periyasamy Mukesh Kumar ${ }^{1, \mathrm{a}}$, Jegadeesan Kumar ${ }^{2}$, Rengasamy Tamilarasan ${ }^{1, \mathrm{~b}}$, \\ Seshachalam Sendhilnathan ${ }^{1}$, and Sivan Suresh ${ }^{3}$ \\ 1 University College of Engineering Pattukkottai (A Constituent College of Anna University) \\ Rajamadam-614 701,Tamilnadu, India \\ 2 Sasurie College of Engineering, Tiruppur-638 056,Tamilnadu, India \\ 3 National Institute of Technology, Tiruchirappalli, Tamilnadu, India \\ E-mail: apcmukeshkumar1975@gmail.com, brrtamilk@yahoo.co.in (Corresponding author)
}

\begin{abstract}
This review paper deals with the nanofluids theoretical thermal conductivity models published by different research groups. It also covers the contrary opinion on anomalous thermal conductivity of nanofluids suggested by different research groups and lists out the nanofluid thermal conductivity models. Numerous works have been carried out on nanofluids and reported the nanofluids have higher thermal conductivity than that of the traditional fluids. In general, the nanofluid theoretical thermal conductivity models have been derived from the classical nanofluid thermal conductivity models. Many research groups proposed the random motion of nanoparticles is the reason for improved thermal conductivity of nanofluids. The thermal conductivity model based on Brownian models is being widely applied in the field of nanofluids application. Though many nanofluids thermal conductivity models have been formulated, there are still some controversial issues covering the inconsistent thermal conductivity data, the sufficiency and suitability of classical nanofluid thermal conductivity models. The nanofluid inconsistencies among the models may be due to the assumption made, depending factors such as particle size, shape, volume fraction, temperature, static, and dynamic conditions of nanoparticles in developing the thermal conductivity models. This review leads to some directions for future research in nanofluids and to help researchers in resolving the controversial issues.
\end{abstract}

Keywords: Nanofluid, effective thermal conductivity, theoretical model, Brownian motion, particle volume fraction.

ENGINEERING JOURNAL Volume 19 Issue 1

Received 24 February 2014

Accepted 30 September 2014

Published 16 January 2015

Online at http://www.engj.org/

DOI:10.4186/ej.2015.19.1.67 


\section{Introduction}

Research and development processes are being carried out for enhancing heat transport properties of traditional heat transfer fluids. The thermal conductivity of heat transfer fluids plays a vital role in the development of energy efficient heat transfer equipment. The conventional heat transfer fluids have already reached their thermal performance limitations. The need for developing novel heat transfer fluids becomes mandatory in order to meet out the high capacity existing cooling requirements. S.U.S. Choi [1] proposed a heat transfer fluids, called nanofluids, as a fluid with suspended nanoparticles in the base fluids and reported the nanofluids have superior thermal properties to the conventional fluids. The nanoparticles may be of metallic, non-metallic and oxides. The base fluid may be of any one of the conventional heat transfer fluids such as Water, Ethylene Glycol, Engine oil etc. In general, nanoparticles have wide scope and potential for applying almost in all fields Farhad Ismail, et al., [2], Sarawut Rimdusit, et al., [3], and Bunjerd Jongsomjit, et al., [4]. Worawut Muangrat, et al., [5], Ekrachan Chaichana, et al., [6]. The potential applications of nanofluids include transportation, electronics cooling, defence, nuclear, space and biomedical as a coolant. Yu, et al., [7] and Jaluria, et al., [8] suggested several factors such as long-term stability, higher pumping power and pressure drop, their thermal performance in turbulent flow, lower specific heat of nanofluids, and higher production cost are the underlining issues in applications of nanofluids.

The special feature of nanofluids in thermal field is the thermal conductivity and much work has been carried out to explore the potential applications of nanofluids. Murshed, et al., [9], Hemanth, et al., [10], Wang and Mujumdar [11], Choi and Routbout[12], and Xiang - Qiwang, et al., [13] described the effect of particle size, shape, concentration of nanoparticles, nanolayer thickness, Brownian motion of particles, and surface charge of particles are the contributors to the anomalous thermal conductivity of nanofluids. They also reviewed the mechanism for enhanced thermal conductivity of nanofluids. Moreover the critical issues in developing the nanofluid are the realistic mechanisms for attaining higher thermal conductivity, settlement of nanoparticles and agglomeration. Rayleigh [14], Jeffrey [15], Tsao [16], and, Yu and Choi [17] have classified the thermal conductivity models based on the arrangement of nanoparticles and found the aggregated path of particles conduct more heat resulting in higher thermal conductivity. Other survey was carried out by Honorine Angue Mintsa, et al., [18] Daxiong Wu, et al., [19] Hiemenz [20], Xuan and Li [21]. They suggested the nanofluid preparation faces challenges such as settling, aggregation, agglomeration, and higher viscosity when particle volume fraction is more. They also suggested the nanofluid thermal conductivity is temperature dependant.

In addition to the research works mentioned above, this paper is aimed at to list out the up-dated nanofluid theoretical thermal conductivity models available in the literature and inconsistency, opinion of researchers working on nanofluids, discrepancy of the published results and the potential for future work.

\section{Theoretical Models for Thermal Conductivity}

\subsection{Modeling Approaches}

There are two approaches commonly used to treat the heat transport properties of mixture and composites Maxwell-Garnett (MG Model) [22]. The first approach proposed by Hui, et al., [23] is the MG model which is applicable only for low particle concentration. The second approach is the Core-Shell Medium (CSM) model which considers the nanoparticle surface. The approach like Rayleigh model takes the effect of particle interaction. The Cichocki-Felderhof (CF) model considered only the interaction between nanoparticles of same radius. The Monecke model rejected the physical topology technique of effective medium theory. This is because of the Monecke model did not consider the effect of clustering. On comparing, the CF model, Monecke model have not been widely used for nanofluid research.

Effective medium and fractal approach are the familiar approach and most of the investigators used this approach to describe the thermal conductivity of particles. The Effective Medium Theory [EMT] is used by Maxwell to explain the thermal conductivity enhancement of slurries and liquid suspensions. Maxwell assumed a very dilute suspension of spherical particles by neglecting the interactions between particles. Moreover Maxwell and EMT approach failed to predict the thermal conductivity of nanofluids with respect to the nanoparticles size and base fluid's temperature. Various researchers like Maxwell [22], Nan, et al., [25], Timofeeva, et al., [26], Williams Evens, et al., [27], and Goa and Zhou [28] used the 
effective medium approach and Wang, et al., [24] used fractal approach. Based on the literature survey, it is studied that the EMT approach is widely used for developing nanofluid thermal conductivity.

Based on the insight view of the particle structure, modelling approach has two types; namely Classical Mechanism Approach (CMA) and Statistical Mechanism Approach (SMA) .In classical mechanism approach there is no insight into the micro structure of nanoparticles. Most of the models used classical mechanism approach for developing the thermal conductivity models. The statistical mechanism has an insight into the molecular space and the basis of intermolecular interactions between the nanoparticles. The first model was developed by Jurij Avsec and Maks Oblak [29] for the thermal conductivity by incorporating the effect of interactions between the nanoparticles. These models were formulated by using Brownian motion and Stokes-Einstein law. The researchers Ren, et al., [30], Bao Yang [31], Jang and Choi [32], Kumar, et al., [33] developed the models based on the kinetic theory approach. Bruggeman model was developed by Hui, et al., [23] by using kinetic theory approach. Jang and Choi [34], Yu and Choi [35], Prasher, et al., [36] and Chandrasekar, et al., [37] formulated the thermal conductivity model based on the convection mode of heat transport. They revealed the effect of nanoconvection caused by the nanoparticles movement in the base fluids.

Therefore most of the classical models have been developed by considering the nanoparticles are in static condition. They developed the thermal conductivity models by treating the nanoparticles are in static condition the heat transfer only by conduction.

\subsection{Combination of Conduction and Convection}

Jang and Choi [32] developed the model by taking the conduction and convection mode of heat transport in nanofluids. Chandrasekar, et al., [37-38] Ravikanth, et al., [39], and Murshed, et al., [40] considered the effect of moving nanoparticles and heat transfer is only by conduction mode. It is observed that the research is carried out on combination of conduction and convection approach for developing nanofluid thermal conductivity. Very little work has been carried out by treating the conduction and convection mode in developing thermal conductivity models

The existing thermal conductivity model has three general categories such as Classical model, Extension of existing conduction models, Development of new models using nanoconvection due to Brownian motion.

\subsection{Classical models}

The models developed to predict the thermal conductivity of well-dispersed solid-liquid mixtures by Maxwell [22], Hamilton-Crosse [41], Bruggeman model [23] and Wasp model [21] are the Classical models. Maxwell [22] model developed to predict the thermal conductivity of micro/millimeter sized particle at low volume fraction. HC model[41] is the modification of Maxwell's model with the inclusion of empirical shape factor $n=3 / \psi$ for spherical and cylindrical medium shapes where, $\psi$ - spherocity defined as the surface area of sphere with the volume equal to that of the particles. It was developed by treating the base fluid and particles as two different phases and well dispersed continuous medium. This is applicable for micrometer and millimeter sized particles. If $\psi$ is equal to one, then the model reduces to Maxwell model [22]. The HC model represents a good description of micrometer or millimeter sized particles. However, the HC model failed to predict the measured thermal conductivity of nanofluid. This model was developed by taking well dispersed discontinuous nanoparticles and continuous base fluids. It has the limitations of spherical shape particles and statistically homogeneous medium. This model is applicable only for the randomly dispersed, uniformly sized non contacting spherical particles. It relies on the continuous medium theory. The classical models deal with the nanofluid thermal conductivity depending parameters such as the particle shape, particles volume fraction, base fluids and particle thermal conductivities. They have been formulated from continuum theory and included only the particle shape and volume fraction $(\% \mathrm{v})$ and it assumes the diffusive heat transfer in both solid and liquid phases.

The Bruggeman model proposed by Hui, et al., [23] is based on mean field approach. The Bruggeman model shows the effect of interaction among the randomly distributed particles and binary homogeneous spherical nanoparticles. It has no limitation of particle volume fraction and it matches well with the Maxwell model at low particle volume fraction. Wasp model was proposed by Xuan and $\mathrm{Li}$ [21] and it is based on macroscopic system model. This model was developed by renovating Hamilton and Crosser model [41] with empirical shape factor equal to one. It fits with the Maxwell model but does not specify the particular shape of the nanoparticles. Wasp model is commonly used for convection heat transfer problems. 
Therefore the classical models are found to be unable to exactly predict the thermal conductivity of nanofluids. This is because the classical models do not include the effect of nanofluid temperature, effect of particle size, interfacial layer of the particle/fluids, particle distribution, nanoparticles cluster, aggregate and Brownian motion of particles into account. Moreover they have been developed by considering the dispersed nanoparticles are in static condition. Therefore the classical models have no insight into the effect of structure, particle size, interfacial layer of the particle/fluids, particle distribution, nanoparticles cluster, aggregate, and Brownian motion.

\subsection{Extension of Conduction Models}

The classical models have considered the effect of nanolayer, interfacial thermal resistance, agglomeration of particles, percolation structure of high-aspect ratio, surface charge state of nanoparticles, cubic arrangement of spherical nanoparticles, and Brownian motion of nanoparticles. This category has two classifications such as static and dynamic model.

\subsubsection{Static model or structural model}

These models were developed by assuming the nanoparticles do not have bulk movement in base fluid and the solid particles in base fluids are composite. The static models were not realistic models and unable to include the effect of particle movement on prediction of thermal conductivity. This is because the nanoparticles are in restless random motion in base fluids. The models (Maxwell [22], Hamilton and Crosser [41] have considered this effect and both are applicable for two phase mixture. Most of the static models were developed by directly modifying Maxwell model and Hamilton and Crosser model [41], or Bruggeman model proposed by Hui, et al., [23]. Maxwell-Garnett's (MG Model). These newly developed models developed by considering the nanoparticles are isolated in base fluid and there is no interaction between the nanoparticles. Moreover these models were developed based on the effective medium theory approach. The nanoparticles and base fluid have been treated by two component systems and they fit well with the Bruggeman classic model. Pak and Cho [42] proposed a thermal conductivity model under the assumptions that the convective heat transfer enhancement is due to mainly the dispersion of suspended nanoparticles in base fluids.

\subsubsection{Inclusion of liquid layering}

Xue [43] was the first to develop the static model for nanofluids based on the liquid layering mechanism and the average polarization theory. Yu and Choi [44], modified the Maxwell model [22], and H.C. model ([41]) included the effect of solid like layer of liquid on the nanoparticles surface which has relatively higher thermal conductivity than the liquid. They replaced the thermal conductivity of nanoparticles with the thermal conductivity of particle and liquid nanolayer. They suggested the solid layer acts as thermal bridge between a solid nanoparticle and a bulk liquid leads to an enhanced thermal conductivity to some extent. They also suggested the positive impact on nanofluids thermal conductivity when the particle diameter is less than $10 \mathrm{~nm}$. This model cannot be applied to the temperature dependent nanofluids to explain the enhanced thermal conductivity and it did not consider any dynamic mechanism. Murshed, et al., [41], expressed their opinion on $\mathrm{Yu}$ and Choi [35], and $\mathrm{Yu}$ and Choi [44] models were considered as not the realistic because the interfacial layer is formed by the molecule wrapping over the particle surface. Yu and Choi [44] derived the simple nanostructure model for the non-structural particles. Sadik kaka and Anchasa Pramuanjaroenkil [46] presented the comparative results of Maxwell model [22], Hamilton and Crosser model [41],Yu and Choi model [35]. They made comparison by taking nanolayer, radius of the nanoparticle, and particle volume fraction into account. The comparisons were made by assuming that the nanolayer thermal conductivity is $5 \mathrm{~W} / \mathrm{mK}$, thickness is $2 \mathrm{~nm}$ and particles size is $15 \mathrm{~nm}$ of $\mathrm{Al}_{2} \mathrm{O}_{3}$ nanoparticles. They suggested all the models have given linear relationship with respect to particle volume fraction. The Maxwell model [22] predicts much lower thermal conductivity ratio than the other two models.

Wang, et al., [24] was the first who developed the nanofluid thermal conductivity model with the aid of fractal model approach and effective medium approximation (EMA) by incorporating the effect of liquid / solid interfacial layer. Murshed, et al., [45] developed a model for cylindrical shaped nanoparticles with the effect of nanolayer thickness into account. They suggested the classical models give underestimated prediction of thermal conductivity of nanofluids. Xie, et al., [47] used the concept of liquid /solid interfacial 
layer to explain the anomalous enhancement of thermal conductivity of nanofluids. Leong, et al., [48] developed a model by considering the interfacial layer as separate component. This was attributed to the inclusion of both static and dynamic mechanism such as particle size, particle movement, particle surface chemistry and interaction potential. Feng, et al. [49], developed a thermal conductivity model which expresses the effective thermal conductivity as a function of nanolayer, the aggregation of nanoparticles, particle size, particle volume fraction, and the thermal conductivity of base fluids. Though many researchers developed thermal conductivity models by incorporating many affecting parameters, Majority among them considered the effect of interfacial liquid layering in developing nanofluids thermal conductivity models.

\subsubsection{Inclusion of interfacial thermal resistance or Kapitza resistance}

The Kapitza resistance exists at the interface of liquid and particles. This effect may be neglected for large grain size materials at room temperature. But for nano-sized structures, the Kapitza resistance can play a significant role in overall thermal performances. Bahrami, et al., [50] suggested the Kapitza resistance will have negative impact on thermal conductivity of nanofluids. Prasher, et al., [36], Ju, et al., [51], and Xue [52] suggested the Kapitza resistance can be incorporated when the effect of nanolayer and interfacial resistance are conside5red for the cylindrical shaped and spherical shaped particles. Jang and Choi [32] considered the effect of Kapitza resistance and found the value is $10^{-2} \mathrm{~m}^{2} \mathrm{~K} / \mathrm{W}$. Keblinski, et al., [53] proposed the thermal conductivity of suspended nanoparticles involving the Kapitza resistance as $k_{\text {nano }}=\beta k_{\text {particle }}$, where $\beta$ is a constant related to Kapitza resistance.

Nan, et al., [25] presented a new nanofluid thermal conductivity model by including the effect of interfacial thermal resistance using the effective medium approach. Murshed, et al., [40] presented a model by assuming the effect of Kapitza resistance is nil. But this assumption is invalid as per the suggestion of Keblinski, et al., [53], Ju, et al., [51], Wang, et al., [24] developed a model of Multi Wall Carbon Nano Tubes (MWCNT) nanofluids by neglecting the Brownian motion of nanotubes and incorporating the effect of Kapitza resistance. They assumed that the MWCNT are randomly oriented.

Murshed, et al., [40] presented a model by incorporating the effect of surface charge, particle size, interfacial layer and Brownian motion. This model has assumed the effect of Kapitza resistance is negligible. $\mathrm{Yu}$ and Choi [56] were the first to develop the model based on the cubic arrangement of spherical nanoparticles with shells. They reported a nonlinear dependence on the particle volume fraction with nanofluid thermal conductivity.

\subsubsection{Dynamic models}

The dynamic models based on the fact that the nanoparticles have lateral and random motion of particles in base fluids along with the effect of nanostructures. This movement of particles leads to the collision and causes micro liquid convection. Therefore the enhanced thermal conductivity of nanofluids is achieved. The movements of nanoparticles are believed to be responsible for enhanced thermal conductivity of nanofluids. The mathematical model for measuring nanoparticles motion velocity is $\mathrm{V}, V \alpha \frac{T^{0.5}}{d_{p}^{1.5}}$ where, $\mathrm{T}$ is the temperature of the fluids and $d_{p}$ is the particle diameter. It shows that the motion velocity is inversely proportional to particle diameter. Xuan, et al., [54] was the first to develop a dynamic model by taking the effects of Brownian motion. This model cannot exactly predict the strong temperature dependence of nanofluids thermal conductivity data obtained by Das, et al., [57], and Patel, et al., [58]. Wang, et al., [59] and Keblinski, et al., [53], developed the model by modifying the Maxwell model. They proposed various mechanisms which were not taken by Maxwell [22], Hamilton and Crosser [41], Hui, et al., [23], and Xuan, et al., [21]. They claimed that the contribution of Brownian motion in nanofluids is not significant. Wang, et al., [59] suggested the microscopic motion of nanoparticles, surface properties and structural effects might be the reason for enhanced thermal conductivity of nanofluids. They also suggested the electric double layer and Vander Walls force could have the strong electro-kinetic effects on the nanoparticles. Keblinski, et al., [53] suggested the four possible mechanisms for improved thermal conductivity of nanofluids. The possible mechanisms are: a) Brownian motion of nanoparticles, b) Nanolayer at the interface of liquid /solid particles, c) Nature of heat transport, and d) The effect of clustering. They reported the thermal diffusion is much faster than the Brownian diffusion and nanoparticles cluster leaves the negative impact 
on the thermal conductivity at low volume concentration. The formation of clustering gives too slow Brownian motion of particles. The formation of cluster also results convection like micro- environment around the particles and due to this heat transfer increases. They suggested the clustering gives both positive and negative impact on increase of thermal conductivity. They concluded that all the possible mechanisms explain the thermal behaviour of nanofluid partially.

Koo and Kleinstreue [60] extended the convection model of Yu, et al., [17] by considering the dragging of fluids by a pair of nanoparticles. Koo and Kleinstreue, [60] claimed that the role of Brownian motion is much more important than that of thermophoretic and osmophoretic motion. They have neglected the interaction of particles at low level concentration $(0.5 \%)$ of particles. Bhattacharya, et al., [61] presented a model based on combining the base fluids and nanoparticle thermal conductivities by replacing $k_{p}$ with effective contribution of the particles. Jang and Choi [32], and Hemanth, et al., [3] expressed their opinion on the enhancement of thermal conductivity of nanofluids.

In particular Hemanth, et al., [10], reported the thermal conductivity enhancement is inversely proportional to particle radius, linearly proportional to particle volume fraction at low volume fraction and temperature dependence is attributed to the variation of Brownian motion of particles. They suggested this model is not used for large volume particle concentration where the inter particles interactions are important. Jang and Choi [32] presented a model which is based on conduction and convection caused by Brownian motion. This model takes the effect of four important modes: a) Collision between the base fluids molecules which represent the base fluids thermal conductivity, b) Thermal diffusion in the base fluids with the effect of Kapitza resistance, c) Collision between the nanoparticles due to Brownian motion, and d) Thermal interactions of dynamic nanoparticles. This model is able to predict the size dependant, temperature dependant and concentration dependant nanofluids. They claimed that the nanoconvection is the key role in enhancing thermal conductivity of nanofluids. Further, Jang and Choi [34] presented sub model by simplifying and adding new concepts of their model Jang and Choi [32]. Jang and Choi [34] incorporated the effect of thermal conductivity of particle, base fluid, volume fraction, particle size, and temperature into account. This new model reports the effect of ratio of thermal conductivity of nanoparticles to that of base fluids is minor in enhancing the thermal conductivity. The model developed by Xuan, et al., [54] contains two terms. The first term represents the contribution due to macroscopic Maxwell model [22], whereas the second term represents the contribution due to Brownian motion of nanoparticles and gives wrong units. They suggested the enhancement of thermal conductivity is by intensification of turbulence due to the presence of nanoparticles. Ren, et al., [30] suggested a thermal conductivity model based on the micro convection, nanolayer, and conduction mode of heat transfer.

Prasher, et al., [36] developed a quasi-empirical Brownian model based on nanoconvection of multiple convections. They reported the nanoconvection caused by the Brownian motion of particles is primary responsible for improved thermal conductivity. They included the effect of convection near the particle and interfacial resistance with unknown parameters. They argued the aggregation of nanoparticles plays a key role in thermal conduction in nanofluids and large cluster tend to settle out which decreases the thermal conductivity. Therefore, the ideas presented by Wang, et al., [59], Keblinski, et al., [53], and Prasher, et al., [36] for enhanced thermal conductivity mechanism are not consistent. This inconsistent may be due to the limitations of particle volume fraction and particle size and shape. Kumar, et al., [33] reported a combined thermal conductivity model by considering the impact of stationary particle and moving particle.

The size effect is taken into account while developing stationary model presented by Xuan [54]. It shows the linear dependence of thermal conductivity with particle volume fraction. The thermal conduction in stationary particle was developed by using the Fourier law of diffusion. The thermal conduction is developed by assuming the two parallel paths of heat flow through the suspension such as heat flow through the liquid particles and heat flow through the solid particles. The moving particle thermal conduction was developed by Stokes-Einstein formula which covers the effect of solid particle radius, liquid particle radius, particle velocity, concentration, fluids temperature, and fluids temperature. They reported that this combined model holds good agreement with gold nanoparticles. This model is suitable for high particle volume concentration. The combined model developed by Kumar, et al., [33] faces more contrary opinion from different research groups. Bastea [62] and Kelblinski, et al., [63] had strong arguments on the combined model. They claimed that the stationary model is unrealistic in consideration of particle radius and liquid radius. They suggested the assumption of mean free path of nanoparticles in dynamic model is unphysical. Chon, et al., [64] presented a model for alumina nanofluids by fitting a curve 
through a linear regression using experimental data. This is the first model to validate the effect of Brownian motion experimentally.

Hyun Kang, et al., [65] formulated a thermal conductivity model for low-particle concentration suspension by using correlation function approach. They derived the lower and upper limits for thermal conductivity of nanofluids. They suggested the shape of the nanoparticles and the agglomeration appreciably influence on the thermal conductivity of nanofluids. They reported the nanofluids thermal conductivity substantially dependents only on the volume fraction of nanoparticles and their characteristics aspect ratio. Gupta, et al., [66] presented a thermal conductivity model by adding the motion of nanoparticles and the motion of liquid molecules in terms of Peclet number. They divided the Brownian motion of nanoparticles into translational and rotational motion. They claimed the Brownian motion of the particles is the most probable mechanism for developing the theoretical models and to explain the anomalous enhancement of thermal conductivity of nanofluids.

\subsubsection{Temperature dependent models}

Das, et al., [57], Patel, et al., [58], Jang and Choi [32], Li, et al., [67], and Murshed, et al. [45] included the effect of temperature in developing the thermal conductivity of nanofluids. They reported the thermal conductivity of nanofluids is temperature dependent. They also suggested the velocity of Brownian motion increases with temperature which leads to enhanced thermal conductivity of nanofluids. A new model was formulated by Ravikanth, et al., [32] which incorporated the Brownian motion effect into account for thermal conductivity of nanofluids along with the functions like temperature, particle size, the properties of nanoparticles and the base fluids. They claimed that their model agree well with the experimental data. However, the thermal conductivity of nanofluids with temperature change is not fully studied experimentally and analytically.

\subsection{Development of New Models Using Nanoconvection due to Brownian Motion}

When a particle immersed in a base fluid, it moves randomly due to the interaction between the particle and its surrounding fluid molecules and this random motion is called "Brownian motion". The Brownian motion of large particles is negligibly small and it is not considered in the traditional particulate flow. The Brownian motion is significant when particle size is in nano level. The newly developed thermal conductivity models are based on the concept of nanoconvection induced by Brownian motion. William Evans, et al., [20] proposed a dynamic simulation model by considering the kinetic theory and effective medium theory of nanoparticles. They showed that there is no significant enhancement due to the Brownian induced hydrodynamic effects. They reported that their simulation model agrees with the experimental results and particle clustering is responsible for anomalous thermal conductivity of nanofluids. Jurij Avsec [29] proposed a thermal conductivity model based on the statistical nanomechanics for very large number of nanoparticles with effective particle volume fractions. They reported that this model holds relatively good agreement with the experimental data. Bao Yang [31] suggested a model by adding the effect of Brownian motion along with the conventional mode called Diffusive conduction. This model was derived from the kinetic theory of fluids by taking into the effect of convection heat transfer caused by the particle Brownian motion. They suggested the long tail of nanoparticle plays a significant role in Brownian motion.

Chandrasekar, et al., [37] proposed two models for predicting thermal conductivity of nanofluids. The one among them is derived from the simple relationship of molecular weight of base fluids and particles proposed by Weber, et al., [68]. They validated their model by experimental data taken from the literature of $\mathrm{Al}_{2} \mathrm{O}_{3}$ / water, $\mathrm{CuO} /$ water, $\mathrm{TiO}_{2}$ /water, and $\mathrm{TiO}_{2}$ / ethylene glycol nanofluids. They reported the first model gives accurate results over a wide range of particle size, particle concentration, different particle materials and base fluids. The second model suggested by Chandrasekar, et al., [38] is based on the model proposed by Shukla, et al., [69] which incorporated the cumulative effect of nanolayer, Brownian motion, particles surface. It has two terms, first term represents the contributions due to macroscopic Maxwell model [22], and which is a special case of HC model ([41]). The second term represents the contribution to Brownian motion of nanoparticles. They revealed the $30 \%$ of enhanced thermal conductivity and this enhancement is due to $21 \%$ by particles shape, $6 \%$ is due to nanolayer thickness and $3 \%$ is due to Brownian motion of particles. They reported the non-spherical shaped particle have greater influence than the spherical shaped particles on the thermal conductivity of nanofluids. However, these models did not 
mention the effect of surface charges and the optimum radius for achieving higher effect of Brownian motion. Li, et al., [67] experimentally investigated with $\mathrm{Al}_{2} \mathrm{O}_{3}$ and $\mathrm{CuO} /$ water based nanofluids. They derived two nanofluid thermal conductivity correlations by incorporating the effect of volume fraction and temperature of base fluids. Chon, et al., [64] presented the relative thermal conductivity model for $\mathrm{Al}_{2} \mathrm{O}_{3}$ nanofluids based on the Reynolds number and Prandtl number. They claimed the relative thermal conductivity of nanofluid increases with higher temperature and smaller diameter of nanoparticles. The review on analytical thermal conductivity of nanofluids by Xiang-Qiwang, et al., [13]. They concluded that there is no reliable model to predict the anomalous thermal conductivity of nanofluids. Lu and Lin [70] suggested a model by considering the near and far field pair interactions of non-spherical nanoparticles.

\subsubsection{Mean free path and Brownian motion model}

Majd Emami Meibodi, et al., [71] developed a model by resistance approach and it covers Brownian motion and interfacial layer along with the particle vibration. They claimed that this model can be used for upper and lower limits of nanofluids without any adjustable parameter. Johnathan J Vadasz, et al., [72] recently proposed the thermal wave via hyperbolic heat conduction effect is the mechanism for enhanced thermal conductivity of nanofluids. They also suggested the hyperbolic heat conduction is the cause for enhanced effective thermal conductivity of nanofluids. Weerapun Duangthongsuk, et al., [73] compared the difference between the measured and computed thermo-physical properties of $\mathrm{TiO}_{2} /$ water nanofluids. They recommended the models proposed by Yu and Choi [35], and Wang, et al., [74] are the most appropriate models for computing the thermal conductivity of nanofluids. Li Yu-Hua, et al., [75] suggested the effect of Brownian motion is not enough to describe the thermal conductivity of temperature dependence nanofluids. Patel, et al., [76] reported the higher specific surface area and Brownian motion are the most significant reasons for the anomalous thermal conductivity of nanofluids. Kaufui Wong, et al., [77] reviewed the heat transfer mechanism in nanofluids and concluded that there is disagreement over several aspects of heat transfer in nanofluids. The mechanism for enhanced nanofluid thermal conductivity is not clear. The influence of particle anisotropy on the effective thermal conductivity of a suspension was experimentally studied by Cherkasova and Shan [78], Chandrasekar, et al., [38] provided an approximation method to determine the heat transfer mechanism for nanofluid thermal conductivity and developed a regime diagram. Keblinski, et al., [63], and Jang and Choi [34] proposed the four factors are responsible for anomalous thermal conductivity. Brownian motion is one of the four factors and they concluded that this effect is very slow as the particle travels long distance to carry heat.

Therefore this review has the scope for investigating the nanofluid thermal conductivity mechanisms and to explain the strange behaviour of nanofluids. They suggested possible factors for enhanced thermal behaviour of nanofluids are based on macro scale and micro scale theory. The macro and micro theory have widely been used by most of the authors. The macro scale possible mechanism includes the heat conduction, particle driven natural convection, conduction induced by electrophoresis, and thermophoresis. The micro scale possible mechanism includes the Brownian motion of particles, liquid-solid interfacial layer, and surface charge state. Most of the investigators developed the models based on the effect of liquid layering and Brownian motion. The models based on Einstein equation dealt only with the particles Brownian motion velocity and temperature. As per the Stokes-law, the spherical particles in the base fluid try to attain sedimentation velocity due to the domination of gravity force. Therefore further work is needed to correlate the sedimentation velocity and Brownian motion velocity for predicting the stability of suspended nanoparticles which determines the effective thermal conductivity. Table 1 shows the list of nanofluids thermal conductivity theoretical models developed.

Table 1. List of nanofluid thermal conductivity theoretical models.

$\begin{array}{lll}\text { Investigator } & \text { Formula } & \text { Comments }\end{array}$

Maxwell [22] $\quad \frac{k_{e f f}}{k_{f}}=\frac{k_{p}+2 k_{f}+2 \phi\left(k_{p}-k_{f}\right)}{k_{p}+2 k_{f}-\phi\left(k_{p}-k_{f}\right)}$

Based on Effective medium theory [EMT], randomly dispersed, and uniformly sized spherical particles. 
Hamilton -

Crosser [41]

$$
\frac{k_{e f f}}{k_{f}}=\frac{k_{p}+(n-1) k_{f}-(n-1) \phi\left(k_{f}-k_{p}\right)}{k_{p}+(n-1) k_{f}+\phi\left(k_{f}-k_{p}\right)}
$$

Bruggeman model

$$
\frac{k_{e f f}}{k_{f}}=\frac{1}{4}\left[(3 \phi-1) \frac{k_{p}}{k_{f}}+(2-3 \phi)+\frac{k_{f}}{4} \sqrt{\Delta}\right]
$$

Hui, X. Zhang, [23]

$$
\Delta=\left[\left[[3 \phi-1]{ }^{2}\left(\frac{k_{p}}{k_{f}}\right)^{2}+(2-3 \phi)^{2}+2\left(2+9 \phi-9 \phi^{2}\right)\left(\frac{k_{p}}{k_{f}}\right)\right]\right]
$$

Wasp model

Xuan and Li

[21]

$$
\frac{k_{e f f}}{k_{f}}=\frac{k_{p}+2 k_{f}-2 \phi\left(k_{f}-k_{p}\right)}{k_{p}+2 k_{f}+\phi\left(k_{f}-k_{p}\right)}
$$

\section{Models derived from Classical models}

Davis [79]

$$
\frac{k_{e f f}}{k_{f}}=1+\frac{3(\alpha-1) \phi}{(\alpha+2)-(\alpha-1) \phi}\left[\phi+f(\alpha) \phi^{2}+0(\phi)^{3}\right]
$$

Maxwell -

Garnett's(MG

Model)[22]

$$
\frac{k_{e f f}}{k_{f}}=\frac{(1-\phi)\left(k_{p}+2 k_{f}\right)+3 \phi k_{p}}{(1-\phi)\left(k_{p}+2 k_{f}\right)+3 \phi k_{f}}
$$

Gupta, et al. [66]

$$
k_{e f f}=k_{f}\left(0.0556 P e+0.1649 P e^{2}-0.0391 P e^{3}+0.0034^{4}\right.
$$

Lu and $\operatorname{Lin}[70]$

$$
\frac{k_{e f f}}{k_{f}}=1+a \phi+b \phi^{2}
$$

Pak and Cho [42]

$$
\frac{k_{e f f}}{k_{f}}=1+7.47 \phi
$$

Wang, et al., [24]

$$
\frac{k_{e f f}}{k_{f}}=\frac{(1-\phi)+3 \phi \int_{0}^{\infty} \frac{k_{c l}(r) n(r)}{k_{c l}(r)+2 k_{f}}}{(1-\phi)+3 \phi \int_{0}^{\infty} \frac{k_{f} n(r)}{k_{c l}(r)+2 k_{f}}}
$$

Xue, et al., [43] $\left(1-\frac{\phi}{\lambda}\right) \frac{k_{\text {eff }}-k_{f}}{2 k_{\text {eff }}+k_{f}}+\frac{\phi}{\lambda}\left[\begin{array}{c}\frac{k_{e f f}-k_{c, x}}{k_{e f f}+B_{2, x}\left(k_{c, x}-k_{\text {eff }}\right)}+4 \\ \frac{k_{\text {eff }}-k_{c, y}}{2 k_{\text {eff }}+\left(1-B_{2, x}\right)\left(k_{c, y}-k_{\text {eff }}\right)}\end{array}\right]=0$
Applicable for spherical and cylindrical particles. Developed by using Shape factor.

For a binary mixture of homogeneous spherical and randomly dispersed nanoparticles.

Particles interaction taken into account. No limitation for particle volume fraction.

Shape factor is unity. Not valid for spherical particles.

Interaction of nanoparticles is taken.

Based on EMT and particles interaction is not taken into account.

Included the effect of translational motion of nanoparticles.

Considered near and far field pair interaction and non-spherical nanoparticles.

Under the assumption that the dispersion of suspended nanoparticles causes the enhancement of thermal conductivity.

Based on effective approximation and fractal theory shows the Brownian motion is not significant.

Based on nanoparticles shells between the particles and base fluids. 
Bu-Xuan

Wang, et al., [24]

Yu and

Choi[35]

Xuan, et al., [54]

$$
\frac{k_{e f f}}{k_{f}}=\frac{k_{p}+2 k_{f}+2 \phi\left(k_{p}-k_{f}\right)}{k_{p}+2 k_{f}-\phi\left(k_{p}-k_{f}\right)}+\frac{\phi \rho c_{p}}{2 k_{f}} \sqrt{\frac{K_{B} T}{3 \pi r_{c} \eta}}
$$

Xie, et al., [52]

$$
\frac{k_{e f f}}{k_{f}}=1+3 \Theta \phi_{T}+\frac{3 \Theta^{2} \phi_{T}^{2}}{1-\Theta \phi_{T}}
$$

Kumar, et al., [33]

$$
\frac{k_{e f f}}{k_{f}}=1+C \frac{2 K_{B} T}{\pi \eta d_{p}^{2}} \times \frac{\phi r_{f}}{k_{f}(1-\phi) r_{p}}
$$

Koo and Kleinstreuer [60]

$$
\frac{k_{e f f}}{k_{f}}=\frac{k_{M G}}{k_{f}}+\frac{1}{k_{f}} \times 5 \times 10^{4} \rho c_{p} \sqrt{\frac{K_{B} T}{\rho D} f(T, \phi)}
$$

Bhattacharya, et al., [61]

$$
k_{e f f}=\phi k_{p}+(1-\phi) k_{f}
$$

Jang and Choi [32]

$$
\frac{k_{e f f}}{k_{f}}=1+C \frac{d_{f}}{d_{p}} k_{f} \phi \operatorname{Re}^{2} d_{p} \operatorname{Pr}
$$

Prasher, et al., [36]

$$
\frac{k_{e f f}}{k_{f}}=\left(1+A \phi \operatorname{Re}^{m} \operatorname{Pr}^{0.333}\right) \frac{(1+2 \alpha)+2 \phi(1-\alpha)}{(1+2 \alpha)-\phi(1-\alpha)}
$$

Chon, et al., [64]

$$
\frac{k_{e f f}}{k}=1+64.7 \phi^{a}\left(\frac{d_{f}}{d_{p}}\right)^{b}\left(\frac{k_{p}}{k_{f}}\right)^{c} \operatorname{Pr}^{d} \operatorname{Re}^{e}
$$

Shukla and Dhir [69]

$$
\frac{k_{e f f}}{k_{f}}=\left[\frac{k_{p}+2 k_{f}+2 \phi\left(k_{p}-k_{f}\right)}{k_{p}+2 k_{f}-\phi\left(k_{p}-k_{f}\right)}\right]+\frac{C \phi(T-T o)}{\mu k a^{4}}
$$

Based on fractal theory and the modification of Bruggeman model. Considered the effect of size, cluster and surface adsorption of particles. Valid for $\mathrm{CuO}$ nanoparticles.

Inclusion of interfacial layer and applied the concept of superposition principle of parallel and series thermal conductivity. Modified Maxwell model and thermal conductivity of equivalent particles taken in to account.

Developed by the random motion of nanoparticles, interfacial interactions and the second term has wrong unit.

Considered the nanolayer thickness.

Based on Fourier law of diffusion and kinetic theory to study the Brownian motion effect

Considered the randomly moving nanoparticles with Brownian motion having unknown parameters.

Inclusion of combined base fluids and nanoparticle thermal conductivities.

Considered the convection and conduction heat transport and dynamic nanoparticles.

Developed by the effect of localized convection near the particle and interfacial resistance.

Relays on Reynolds number based on Brownian and $\mathrm{Al}_{2} \mathrm{O}_{3}$ nanoparticles.

Based on Macroscopic model, Brownian motion and set the lower limit for Brownian motion. 


\section{Comments}

Yu and Choi [44]

$$
\frac{k_{e f f}}{k}=1+\frac{n \phi A}{1-\phi A}, \mathrm{~A}=\frac{1}{3} \sum_{i=a, b, c} \frac{k_{p j}-k_{f}}{k_{p j}+(n-1) k_{f}}
$$

Inclusion of interfacial layer and applied the concept of superposition principle of parallel and series thermal conductivity. Modified HC model with $n=3 \psi^{-\alpha}$.

Based on homogeneous distribution of nanoparticles.

Included the effect of static and dynamic mechanism. Considered interfacial layer as separate component and for spherical nanoparticles.

$$
\frac{k_{e f f}}{k_{f}}=1+0.764481 \phi+0.01868867 T-0.46214175
$$

$\mathrm{Li}$ and For Al2O3/water nanofluids

Peterson [67]

$$
\frac{k_{e f f}}{k_{f}}=1+3.76108 \phi+0.017924 T-0.30734
$$

\section{For CuO/water nanofluids}

William Evans, et al., [27]

Goa [28]

$$
1-\phi=\left(\frac{k_{f}}{k_{e f f}}\right)^{3 A}\left(\frac{k_{f}+B_{1}}{k_{e f f}+B_{1}}\right)^{3 C_{1}}\left(\frac{k_{f}+B_{2}}{k_{e f f}+B_{2}}\right)^{3 C_{2}}
$$

Jang and Choi [34]

$$
k_{e f f}=k_{f}(1-\phi)+\beta k_{p} \phi+\phi h \delta_{T}
$$

Jurij Avsec

$$
\frac{k_{e f f}}{k_{f}}=\left[\frac{k_{p}+(n-1) k_{f}-(n-1) \alpha_{e}\left(k_{f}-k_{p}\right)}{k_{p}+(n-1) k_{f}+\alpha_{e}\left(k_{f}-k_{p}\right)}\right]
$$

Based on statistical nanomechanics and consideration of liquid layer thickness.

Based on EMT for $\mathrm{Al}_{2} \mathrm{O}_{3}$ nanofluids with the effect of agglomeration. 
Murshed, et $\quad k_{\text {eff }}=\left[\begin{array}{l}\left(k_{p}-k_{l r}\right) \phi k_{l r}\left[2 \gamma_{1}^{3}-\gamma^{3}+1\right]+\left(k_{p}+2 k_{l r}\right) \gamma_{1}^{3} \\ {\left[\phi \gamma^{3}\left(k_{l r}-k_{f}\right)+k_{f}\right]}\end{array}\right]$ al., [45]

$$
\left[\left(\gamma_{1}^{3}\left(k_{p}+2 k_{l r}\right)-\left(k_{p}-k_{l r}\right) \phi\left[\gamma_{1}^{3}+\gamma^{3}-1\right]\right]\right.
$$

Bao Yang [31]

$$
\frac{k_{e f f}}{k_{f}}=1+3 \phi \frac{\alpha-1}{\alpha+2}, \text { where } \alpha=\frac{r_{p}}{R_{b} k_{f}}
$$

Leonid

Braginsky, et

al., [80]

Chandrasekar,
et al., [37]

Ravikanth, et al., [39]

$$
\begin{gathered}
k_{\min }^{-1}=\phi k_{s}^{-1}+(1-\phi) k_{l}^{-1} \\
k_{\max }=\phi k_{s}+(1-\phi) k_{l}
\end{gathered}
$$

Considered the interfacial layer and base fluid temperature.

Formulated by taking the diffusive conduction and Brownian motion into account. Based on kinetic theory of nanoparticles.

Relays on Correlation function approach and considered the characteristics' aspect ratio.

Fits well with low particle volume fraction.

Developed by macroscopic model of $\mathrm{HC}$ and inclusion of Brownian motion with respect to temperature.

Applicable for the particle range of 29-77nm.Based on Classical model and Brownian motion of particles.

Combination of Static and dynamic mechanism. Developed by Modifying the Brownian motion and DLVO potential.

Formulated by particle resistance approach and Mean free path of nanoparticles.

Based on the prediction of thermal conductivity of water and the molecular weight of particle and base fluids.

Comprehensive model involving nanoparticle size, nanolayer thickness, the interaction of adjacent nanoparticles, volume fraction and temperature. 


\section{Conclusions}

This paper covers the review on nanofluids thermal conductivity theoretical models, different approaches in developing thermal conductivity models, affecting parameters, mechanisms for enhanced thermal conductivity, and contrary opinion on nanofluids thermal conductivity. The thermal conductivity of a nanofluid depends on many parameters such as base fluids, nanoparticle volume fraction, particle size, shape, temperature, surface charge, $\mathrm{pH}$ value, particle materials, Brownian motion of nanoparticles, effect of clustering, nanolayer, and dispersion techniques. The experimental results show that the thermal conductivity of nanofluid did not agree with the theoretical models results. The deviation between the theoretical model and experimental results may be due the effect of above said depending factors. Most of the reports on nanofluid thermal conductivity state that the thermal conductivity increases with increasing nanoparticles volume fraction. But up to what particle volume fraction the thermal conductivity of nanofluids increase is not clear. Therefore, there is a scope to investigate the limit of nanoparticle volume fraction in thermal conductivity enhancement. Moreover, most of the models deal with the Brownian motion of nanoparticles. But none of the model relates the Brownian motion velocity and settling velocity and optimum particle size for attaining the stability of nanofluids. It is seen from the literature that there is no report on the optimum level of nanoparticles to be dispersed in the base fluids for attaining stability and less tendency to agglomerate. Moreover there is no report on the critical size to attain stability and low aggregate formation. Further work is needed to optimize the particle size and particle volume fraction, and temperature factors for developing new thermal conductivity models for attaining optimum thermal conductivity of nanofluids.

\section{Symbols \\ Nomenclature}

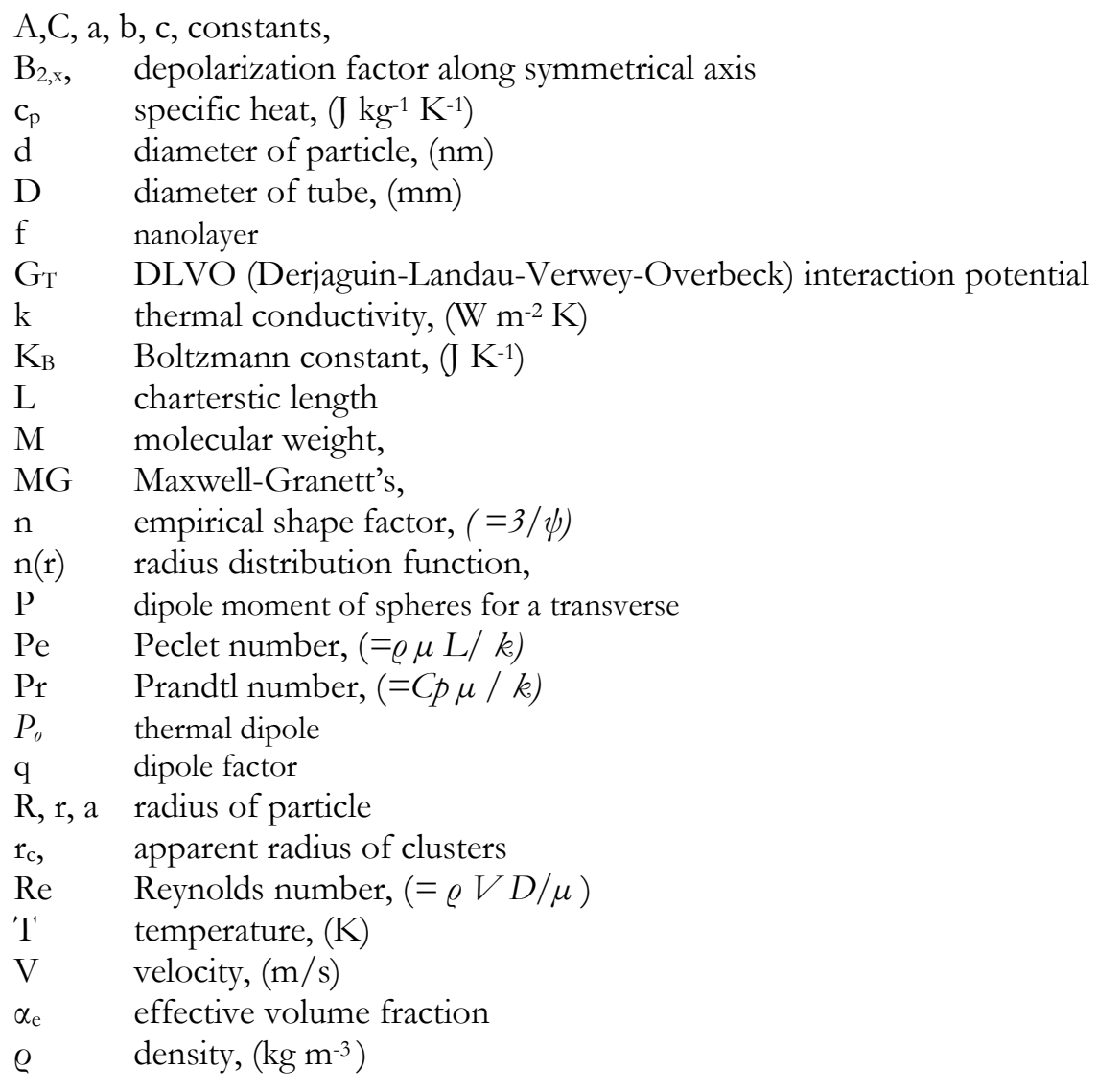

\section{Greek symbols}

$\mu \quad$ dynamic viscosity 
$\eta \quad$ intrinsic viscosity

$\alpha \quad$ aspect ratio of nanoparticles

$\phi_{T} \quad$ modified total volume fraction of the original nanoparticle and nano-layer

$\gamma \quad$ ratio of nanolayer thermal conductivity to particle thermal conductivity, $\left(=k_{\text {layer }} / k_{p}\right)$

$\beta \quad$ ratio of nanolayer thickness to the original particle radius, $(=h / r)$

$\Lambda \quad$ thermal conductivity of suspension regardless of particle motion and vibration

$\delta \quad$ thickness of interfacial layer

$\phi \quad$ volume fraction

$c_{\mathrm{p}} \quad$ compound particle

\section{Subscripts}

$\begin{array}{ll}\mathrm{b}, 1, \mathrm{f} & \text { base fluid } \\ \mathrm{cl} & \text { cluster of particles } \\ \mathrm{eff} & \text { effective } \\ \mathrm{lr} & \text { liquid layer } \\ \mathrm{nf} & \text { nanofluid } \\ \mathrm{p} & \text { nanoparticle }\end{array}$

\section{References}

[1] S. U. S. Choi, "Developments and applications of non-newtonian flow," ASME FED, vol. 66, pp. 99-105, 1995.

[2] F. Ismail, A. I. Rashid, and M. Mahbub, and "CFD analysis for optimum thermal design of carbon, nanotube based micro-channel heat sink," Engineering Journal, vol. 15, no. 4, 2011.

[3] S. Rimdusit, K. Punson, I. Dueramae, A. Somwangthanaroj, and S. Tiptipakorn, "Rheological and thermomechanical characterizations of fumed silica-filled polybenzoxazine nanocomposites," Engineering Journal, vol. 15, no. 3, 2011.

[4] B. Jongsomjit, K. PinKaew, O. Mekasuwandumrong, J. Panpranot, A. Shotipruk, P. Praserthdam, and J. G. Goodwin Jr., "zirconia modification on nanocrystalline titania-supported cobalt catalysts for methanation,” Engineering Journal, vol. 16, no. 4, 2012.

[5] W. Muangrat, S. Porntheeraphat, and W. Wongwiriyapan, "Effect of metal catalysts on synthesis of carbon nanomaterials by alcohol catalytic chemical vapor deposition," Engineering Journal, vol. 17, no. 5, 2013.

[6] E. Chaichana, S. Pathomsap, T. Shiono, and B. Jongsomjit, "Observation of bimodal LLDPE/ $\mathrm{TiO}_{2}$ nanocomposites produced by in situ polymerization with zirconocene/MMAO catalysts via Ga modification on $\mathrm{TiO}_{2}$ nanofiller," Engineering Journal, vol. 17, no. 3, 2013.

[7] W. Yu, D. M. France, S. U. S. Choi, and J. L. Routbort, "Review and assessment of nanofluid technology for transportation and other applications," Argonne National Laboratory, April 2007.

[8] Y. Jaluria, O. Manca, D. Poulikakos, K.Vafai, and L. Wang, "Heat transfer in nanofluids 2012," Advances in Mechanical Engineering, vol. 4, 2012. doi: 10.1155/2012/972973

[9] S. M. S. Murshed, K. C. Leong, and C. Yang, "Investigations of thermal conductivity and viscosity of nanofluids," International Journal of Thermal Sciences, vol. 47, pp. 560-568, 2008.

[10] K. D. Hemanth, H. E.Patel, K. V. R. Rajeev, T. Sundrarajan, T. Pradeep, and S. K. Das, "Model for heat conduction in nanofluids," Physical Review Letters, vol. 93, no. 14, pp. 144301, 2004.

[11] X. Q. Wang and A. S. Mujumdar, "Heat transfer characters of nanofluids: A review," International Journal Thermal Sciences, vol. 46, pp. 1-19, 2007.

[12] W. Yu, D. M. France, S. U. S. Choi, and J. L. Routbout, "Review and assessment of nanofluids technology for transportation and other applications," Argonne National Laboratory (ANL), (No. ANL/ESD/07-9, 2007.

[13] Q. Xiang and S. M. Arun, "A review on nanofluids-part I: Theoretical and numerical investigations," Brazilian Journal of Chemical Engineering, vol. 25, no. 4, pp. 613-630, 2008.

[14] L. Rayleigh, "On the influence of obstacle arranged in rectangular order upon the properties of medium," Philo Magazine, vol. 34, pp. 481-502, 1892. 
[15] D. J. Jeffrey, "Conduction through a random suspension of spheres," London: Proc. R. Soc, vol. 335, no. 1602, pp. 355-367, 1973.

[16] G. T. N. Tsao, "Thermal conductivity of two-phase materials," Ind. Eng. Chem, vol. 53, no. 5, pp. 395397, 1961.

[17] W. Yu and S. U. S. Choi, "The role of interfacial layers in the enhanced thermal conductivity of nanofluids a renovated Maxwell model," Journal of Nanoparticles, vol. 6, pp. 167-71, 2003.

[18] A. M. Honorine, R. Gilles, C. T. Nguyen, and D. Dominique, "New temperature dependent thermal conductivity data of water based nanofluids," International Journal of Thermal Sciences, vol. 48, pp. 363371, 2009.

[19] W. Daxiong, Z. Haitao, W. Liqiu, and L. Liu, "Critical issues in nanofluids preparation, charactererization and thermal conductivity," Current Science, vol. 5, pp. 103-112, 2009.

[20] P. C. Hiemenz, Principles of Colloid and Surface Chemistry. New York: Marcel Dekker, 1986.

[21] Y. Xuan, and Q. Li, "Heat transfer Enhancement of nanofluids," International Journal of Heat and Fluids Flow, vol. 21, pp. 58-64, 2000.

[22] J. C. Maxwell, A Treatise on Electricity And Magnetism. UK: Clarendon, 1973.

[23] P. M. Hui, X. A. Zhang, J. Markworth, and D. Stroud, "Thermal conductivity of graded composites: Numerical simulations and an effective medium approximation," Journal of Materials Sciences, vol. 34, pp. 5497-5503.1999.

[24] B. X. Wang, L. P. Zhou, and X.F. Peng, "Fractal model for predicting the effective thermal conductivity of liquid with suspension of nanoparticles," International Journal of Heat and Mass Transfer, vol. 46, pp. 2665-2672, 2003.

[25] C. W. Nan, Z. Shi, and Y. Lin, "A simple model for thermal conductivity of carbon nanotubes-based composites," Chemical Physics Letters, vol. 375, pp. 666-669, 2003.

[26] E. Y. Timofeeva, A. N. Gavrilov, J. M. McCloskey, and Y. V. Tolmachev, "Thermal conductivity and particle agglomeration in alumina nanofluids: Experiment and theory,” Physical Review, vol. 76, no. 6, 2007.

[27] W. Evans., J. Fish, and P. Keblinski, "Role of Brownian motion hydrodynamics on nanofluids thermal conductivity," Applied Physics Letters, vol. 88, 2006.

[28] L. Goa and X. F. Zhou, "Differential effective medium theory for thermal conductivity in nanofluids," Physics Letters A, vol. 348, no. 3, pp. 355-360, 2006.

[29] J. Avsec and M. Oblak, "The calculation of thermal conductivity, viscosity and thermodynamics," International Journal of Heat and Mass Transfer, vol. 50, pp. 4331-4341, 2007.

[30] Y. S. Ren, H. Xie, and A. Cai, "Effective thermal conductivity model of nanofluids containing spherical nanoparticles", Journal of Physics D: Applied Physics, vol. 38, pp. 3958-3961, 2005.

[31] B. Yang, "Thermal conductivity Equations based on Brownian motion in suspensions of nanoparticles (nanofluids)," Journal of Heat Transfer, vol. 130, no. 4, 2008.

[32] S. P. Jang and S. U. S. Choi, "Role of Brownian motion in the enhanced thermal conductivity of nanofluids," Applied Physics Letters, vol. 84, no. 21, pp. 4316-4318, 2004.

[33] D. H. Kumar, H. E. Patel, V. R. R. Kumar, T. Sundararajan, T. Pradeep, and S. K. Das, "Model for heat conduction in nanofluids," Physical Review Letters, vol. 93, no. 14, 2004.

[34] S. P. Jang and S. U. S. Choi, "Effect of various parameters on nanofluids thermal conductivity," Transaction of ASME, Journal of Heat Transfer, vol. 129, pp. 617-623, 2007.

[35] W. Yu and S. U. S. Choi, "The role of interfacial layer in the enhanced thermal conductivity of nanofluids: A renovated Maxwell model," Journal of Nanoparticle Research, vol. 5, pp. 167-171, 2003.

[36] R. Prasher, P. Bhattacharya, and P.E. Phelan, "Thermal conductivity of nanoscale colloidal solutions nanofluids," Physics Review Letter, vol. 94, 2005.

[37] M. Chandrasekar, S. Suresh, R. Srinivasan, and A. Chandra bose, "New analytical models to investigate thermal conductivity of nanofluids," Journal of Nanoscience and Nanotechnology, pp. 533-538, 2009.

[38] M. Chandrasekar and S. Suresh, "Determination of heat transfer mechanism in aqueous nanofluids using regime diagram," Chinese Physics Letters, vol. 26, no. 12, pp. 4401, 2009.

[39] S. Ravikanth, S. Vajjha, and K. Dedendra, "Experimental determination of thermal conductivity of nanofluid and development of new correlation," International Journal of Heat and Mass Transfer, vol. 52, pp. 4675-4682, 2009.

[40] S. U. S. Murshed, K. C. Leong, and C. Yang, "A combined model for the effective thermal conductivity of nanofluids," Applied Thermal Engineering, vol. 29, pp. 2477-2483, 2009. 
[41] R. L. Hamilton and O. K. Crosser, "Thermal conductivity of heterogeneous two-component systems," IEC Fundamental, vol. 1, no. 3, pp. 187-191, 1962.

[42] B. C. Pak and Y. I. Choi, "Hydraulic and heat transfer study of dispersed fluids with submicron metallic oxide particles," Experimental Heat Transfer, vol. 11, pp. 151-170, 1998.

[43] Q. Z. Xue, "Model for effective thermal conductivity of nanofluids," Physics Letters, pp. 313-317, 2003.

[44] W. Yu and S. U. S. Choi, "The role of interfacial layer in the enhanced thermal conductivity of nanofluids: a renovated Hamilton-Crosser model," Journal of Nanoparticle Research, vol. 6, pp. 355-361, 2004.

[45] S. M. S. Murshed, K. C. Leong, and C. Yang, "Thermo-physical and electro kinetic properties of nanofluids-A critical review," Applied Thermal Engineering, vol. 28, pp. 2109-2125, 2008.

[46] S. Kakaç and A. Pramuanjaroenkij, "Review of convective heat transfer enhancement with nanofluids," International Journal Heat and Mass Transfer, vol. 52, pp. 3187-3196, 2009.

[47] H. Xie, M. Fujii, and X. Zhang, "Effect of interfacial nanolayer on the effective thermal conductivity of nanoparticle-fluid mixture," International Journal of Heat and Mass Transfer, vol. 48, pp. 2926-2932, 2005.

[48] K. C. Leong, C. Yang, and S. M. S. Murshed, "A model for the thermal conductivity of nanofluids-the effect of interfacial layer," Journal of Nanoparticles Research, vol. 8, pp. 245-254, 2006.

[49] Y. Feng, B. Yu, P. Xu, and M. Zou, "The Effective conductivity of nanofluids based on the nanolayer and the aggregation of nanoparticles," Journal of Physics D;Applied Physics, vol. 40, pp. 3164-3171, 2007.

[50] M. Bahrami, M. M. Yovanovich, and J. R. Culham, "Assessment of relevant physical phenomena controlling thermal performance of nanofluids," in Proceedings of IMECE, ASME, Illinois, USA, November 5-10, 2006.

[51] S. Ju and Z. Y. Li, "Theory of thermal conductance in carbon Nanotube composites," Physics Letters, vol. 335, pp. 335-367, 2006.

[52] Q. Z. Xue, "Model for the effective thermal conductivity of carbon Nanotube composites," Journal of Nanotechnology, vol. 17, pp. 1655-1660, 2006.

[53] P. Keblinski, S. R. Phillpot, S. U. S. Choi, and J. A. Eastman, "Mechanisms of heat flow in suspensions of nanosized particles nanofluids," Interactional Journal of Heat and Mass Transfer, vol. 45, pp. 855-863, 2002.

[54] Y. Xuan, Q. Li, and W. Hu, "Aggregation structure and conductivity of nanofluids," AICHE Journal, vol. 49, no. 4, pp. 1038-1043, 2003.

[55] H. E. Patel, K. B. Anoop, T. Sundrarajan, and S. K. Das, "Model for thermal conductivity of CNT nanofluids," Bulletin of Materials Science, vol. 31, no. 3, pp. 387-390, 2008.

[56] W. Yu and S. U. S. Choi, "An effective thermal conductivity model of nanofluids with a cubic arrangement of spherical particles," Journal of Nanoscience and Nanotechnology, vol. 5, pp. 580-586, 2005.

[57] S. K. Das, N. Putra, P. Thesien, and W. Roetzel, "Temperature dependence of thermal conductivity enhancement for nanofluids," Journal of Heat Transfer, vol. 125, pp. 567-574, 2003.

[58] H. E. Patel, S. K. Das, T. Sundrararajan, N. A. Sreekumaran, B. George, and T. Pradeep, "Thermal conductivities of naked and monolayer protected metal nanoparticles based nanofluids: manifestation of anomalous enhancement and chemical effects," Applied physics Letters, vol. 83, no. 14, pp. 2931 2933, 2003.

[59] X. Wang, X. Xu, and S. U. S. Choi, “Thermal conductivity of nanoparticles-fluid mixture," Journal of Thermo Physics and Heat Transfer, vol. 13, pp. 474-480, 1999.

[60] J. Koo and C. Kleinstreuer, "Impact analysis of nanoparticles motion mechanisms on the thermal conductivity of nanofluids," International Communications in Heat and Mass Transfer, vol. 32, no.9, pp. 1111-1118, 2005.

[61] P. Bhattacharya, S. K. Saha, A. Yadav, P. E. Phelan, and R.S. Prashar, "Brownian dynamics simulation to determine the effective thermal conductivity of nanoparticles," Journal of Applied Physics, vol. 95, pp. 6492-6494, 2004.

[62] S. Bastea, "Comment on Model for heat conduction in nanofluids," Physics Review Letter, vol. 95, no. 1, 2007.

[63] P. Keblinski and D. G. Cahill, "Comment on model for heat conduction in nanofluids," Physics Review Letters, vol. 20, pp. 9401-9520, 2005. 
[64] C. H. Chon, K. D. Kihm, S. P. Lee, and S. U. S. Choi, "Empirical correlation finding the role of temperature and particles size of nanofluids $\left(\mathrm{Al}_{2} \mathrm{O}_{3}\right)$ Thermal conductivity enhancement," Applied Physics Letters, vol. 87, pp. 1-3, 2005.

[65] U. Hyun, K. Kang, K. Wun-Gwi, and S. H. Kim, "Effect of particle migration on the heat transfer of nanofluids," Korea-Australia Rheology Journal, vol. 19, no. 2, pp. 99-107, 2007.

[66] S. K. Gupta, S. G. Advani, and P. Huq, "Role of Micro-convection due to nonaffine motion of particle in mono-disperse suspension," International Journal of Heat and Mass Transfer, vol.38, no. 15, pp. 2945-2958, 1995.

[67] C. Li and G. P. Peterson, "Experimental investigation of temperature and volume fraction variations on the effective thermal conductivity of nanoparticles suspensions (nanofluids)," Journal of Applied Physics, vol. 99, no. 8, 2006.

[68] H. F. Weber, L. A. Wenzel, C. W. Clamp, L. Maus, and L.B. Andersen, Principles of Unit Operations. John Wiley \& Sons, 2008.

[69] R. K. Shukla and V. K. Dhir, "Study of the effective thermal conductivity of nanofluids," in Proc. ASME 2005 International Mechanical Engineering Congress and Exposition, pp. 537-541.

[70] S. Lu and H. Lin, "Reflective conductivity of composite containing aligned spherical inclusions of finite conductivity," Journal of Applied Physics, vol. 79, pp. 6761-6769, 1996.

[71] M. E. Meibodi, M. Vafaie-Sefti, A. M. Rashidi, A. Amrollahi, M. Tabasi, and H. S. Kalal, "Simple model for thermal conductibility of nanofluids using resistance model approach," International Communications in Heat and Mass Transfer, vol. 37, no. 5, pp. 555-559, 2010.

[72] J. V. Johnathan and G. Saneshan, "Thermal wave effects on heat transfer enhancement in nanofluids suspensions," International Journal of Thermal Science, vol. 49, pp. 235-242, 2010.

[73] D. Weerapun and W.S omachi, "Comparison of the effects of measured and computed thermo physical properties of nanofluids on heat transfer performance," Experimental Thermal Fluid Science, vol. 34, pp. 616-624, 2010.

[74] X. Q. Wang and A. S. Mujumdar, "Heat transfer characters of nanofluids: A review," International, Journal Thermal Science, vol. 46, pp. 1-19, 2008.

[75] L. Yu-Hua, Q. Wei, and F. Jian-Chao, "Temperature dependence of thermal conductivity of nanofluids," Chinese Physics Letters, vol. 25, no. 9, pp. 3319, 2008.

[76] H. E. Patel, T. Sundrarajan, T. Pradeep, A. Dasgupta, N. Das Gupta, and S. K. Das, "A microconvection model for thermal conductivity of nanofluids," Pramana-Journal of Physics, vol. 65, no. 5, pp. 863-869, 2005.

[77] V. W. Kaufui and C. Michael, "Heat transfer mechanisms of clustering in nanofluids," Advances in Mechanical Engineering, 2010. doi: 10.1155/2010/795478

[78] A. S. Cherkasova and J. W. Shan, "Particle aspect ratio effects on thermal conductivity of micro and nanoparticle suspensions," Journal of Heat Transfer, vol. 130, no. 8, 2008.

[79] R. H. Davis, "The viscosity of suspensions of spherical particles," Recueil des travaux chimiques des PayesBas, vol. 61, no. 863-87, 1942.

[80] B. Leonid and S. Valery, "Thermal conductivity of low-particle concentration suspensions: Correlation function approach," Physical Review Letter B, vol. 78, no. 22, 2008.

[81] M. Chandrasekar, S. Suresh, and A. Chandra Bose, "Experimental investigations and theoretical determination of thermal conductivity and viscosity of $\mathrm{Al}_{2} \mathrm{O}_{3}$ /water nanofluids," Experimental Thermal and Fluids Science, vol. 34, pp. 210-216, 2010.

[82] W. Wang, L. Lin, Z. X. Feng, and S. Y. Wang, "A comprehensive model for the enhanced thermal conductivity of nanofluids," Journal of Advanced Research in Physics, vol. 3, no. 2, 2012. 
